\title{
Quantitative analysis of late iodine enhancement using dual-layer spectral detector computed tomography: comparison with magnetic resonance imaging
}

\author{
Peijun Liu ${ }^{1}$, Lu Lin ${ }^{1}$, Cheng Xu', Yechen $\mathrm{Han}^{2}$, Xue Lin ${ }^{2}$, Yang Hou ${ }^{3}$, Xiaomei Lu ${ }^{4}$, Mani Vembar \\ Zhengyu Jin ${ }^{1}$, Yining Wang ${ }^{1}$ \\ ${ }^{1}$ Department of Radiology, State Key Laboratory of Complex Severe and Rare Diseases, Peking Union Medical College Hospital, Chinese Academy \\ of Medical Sciences and Peking Union Medical College, Beijing, China; ${ }^{2}$ Department of Cardiology, State Key Laboratory of Complex Severe and \\ Rare Diseases, Peking Union Medical College Hospital, Chinese Academy of Medical Sciences and Peking Union Medical College, Beijing, China; \\ ${ }^{3}$ Department of Radiology, Shengjing Hospital of China Medical University, Shenyang, China; ${ }^{4}$ Clinical Science, Philips Healthcare, Beijing, China; \\ ${ }^{5}$ CT Clinical Science, Philips Healthcare, Cleveland, OH, USA
}

Contributions: (I) Conception and design: Y Wang, Z Jin; (II) Administrative support: Y Hou; (III) Provision of study materials or patients: Y Han, X Lin; (IV) Collection and assembly of data: P Liu, C Xu; (V) Data analysis and interpretation: P Liu, L Lin, X Lu, M Vembar; (VI) Manuscript writing: All authors; (VII) Final approval of manuscript: All authors.

Correspondence to: Yining Wang; Zhengyu Jin. Department of Radiology, State Key Laboratory of Complex Severe and Rare Diseases, Peking Union Medical College Hospital, Chinese Academy of Medical Sciences and Peking Union Medical College, No. 1, Shuaifuyuan, Dongcheng District, Beijing 100730, China. Email: wangyining@pumch.cn; jinzy@pumch.cn.

Background: To evaluate the segmental myocardial extracellular volume (ECV) fraction and to define a threshold ECV value that can be used to distinguish positive late gadolinium enhancement (LGE) segments from negative myocardial segments using dual-layer spectral detector computed tomography (SDCT), with magnetic resonance imaging (MRI) as a reference.

Methods: Fifty-six subjects with cardiac disease or suspected cardiac disease, underwent both late iodine enhancement on CT (CT-LIE) scanning and late gadolinium enhancement on MRI (MRI-LGE) scanning. Each procedure occurred within a week of the other. Global and segmental ECVs of the left ventricle were measured by CT and MRI images. According to the location and pattern of delayed enhancement on MRI image, myocardial segments were classified into 3 groups: ischemic LGE segments (group 1), nonischemic LGE segments (group 2) and negative LGE segments (group 3). The correlation and agreement between CT-ECV and MRI-ECV were compared on a per-segment basis. Receiver operating characteristic (ROC) curve analysis was performed to establish a threshold for LIE detection.

Results: Among the 56 patients, 896 segments were analyzed, and of these, 73 segments were in group 1, 229 segments were in group 2, and 594 segments were in group 3. In segmental analysis, CT-ECV in group $3(27.0 \% ; 24.9-28.9 \%)$ was significantly lower than that in group $1(33.2 \% ; 30.7-36.3 \%)$ and group $2(34.9 \%$; 32.3-39.8\%; all $\mathrm{P}<0.001)$. Good correlations were seen between CT-ECV and MRI-ECV for all groups (group 1: r=0.920; group 2: $\mathrm{r}=0.936$; group 3: $\mathrm{r}=0.799$; all $\mathrm{P}<0.001$ ). Bland-Altman analysis between CTECV and MRI-ECV showed a small bias in all 3 groups (group 1: $-2.1 \%, 95 \%$ limits of agreement $-11.3-$ $7.1 \%$; group 2: $-0.6 \%, 95 \%$ limits of agreement $-13.1-11.9 \%$; group $3: 1.0 \%, 95 \%$ limits of agreement -12.7-14.7\%). CT-ECV could differentiate between LGE-positive and LGE-negative segments with $83.1 \%$ sensitivity and $93.3 \%$ specificity at a cutoff of $31 \%$.

Conclusions: ECV values derived from CT imaging showed good correlation and agreement with MR imaging findings, and CT-ECV provided high diagnostic accuracy for discriminating between LGE-positive and LGE-negative segments. Thus, cardiac CT imaging might be a suitable noninvasive imaging technique for myocardial ECV quantification. 
Keywords: Extracellular volume fraction (ECV fraction); spectral detector computed tomography (SDCT); myocardial delayed enhancement

Submitted Mar 29, 2021. Accepted for publication Jun 25, 2021.

doi: 10.21037/qims-21-344

View this article at: https://dx.doi.org/10.21037/qims-21-344

\section{Introduction}

Myocardial extracellular volume (ECV) fraction with magnetic resonance imaging (MRI) is now the reference standard for the noninvasive assessment of myocardial fibrosis (1-4). Myocardial ECV is elevated in a variety of cardiomyopathies, including both ischemic and nonischemic cardiomyopathy $(5,6)$. However, cardiac MRI examinations are not appropriate for patients with claustrophobia or metallic implants.

Recently, ECV quantification derived from CT has been reported in several studies with a good correlation between CT-ECV and MRI-ECV (7-11). Iodine density images derived from dual-energy CT represent distribution of iodine in a voxel in a state of equilibrium. After contrast injection, ECV by cardiac CT is calculated from an iodine density map (incorporating the patient's hematocrit level), thus reducing the possibility of misregistration between the pre- and postcontrast CT images and improving measurement accuracy (10). A previous study also demonstrated a threshold value of CT-ECV that distinguishes healthy myocardium from cardiomyopathy (12). However, ECV assessment among ischemic, nonischemic, and late gadolinium enhancement (LGE) negative myocardial segments using dual-layer spectral detector CT has not been studied.

The aim of this study was to evaluate segmental myocardial ECV fraction and to define a threshold ECV value to distinguish LGE-positive segments from LGEnegative myocardial segments using dual-layer spectral detector CT with MRI as a reference.

\section{Methods}

The study was conducted in accordance with the Declaration of Helsinki (as revised in 2013). This study was approved by the Institutional Committee of Ethics of Peking Union Medical College Hospital. All participants signed written informed consent before cardiac CT and MRI scans.

\section{Study population}

In this prospective study, consecutive patients diagnosed with cardiac disease or with suspected cardiac disease underwent both late iodine enhancement (LIE) on CT (CT-LIE) and MRI-LGE on separate occasions within a week from each other between December 2018 and December 2020. Patient exclusion criteria included allergy to iodine and gadolinium contrast agents, insufficient renal function, claustrophobia, non-MRI compatible devices (such as metallic implants), age $<20$ years, and previous cardiac surgery, or pregnancy. In total, 126 patients with cardiac disease or with suspected cardiac disease were initially included. The flowchart of population enrollment is showed in Figure 1.

\section{Cardiac CT examination}

Cardiac CT was performed with a dual-layer spectral CT scanner (IQon, Philips Healthcare, Best, the Netherlands). Patients received oral $\beta$-blockers 1 hour before CT scanning when the heart rate was $>70$ beats $/ \mathrm{min}$. All patients received sublingual nitroglycerin $3 \mathrm{~min}$ before scanning and were given breath-holding instructions before scanning.

Coronary CT angiography was performed using a prospective axial scanning mode if the patients had a heart rate $<70$ beats/min or a retrospective helical scanning mode if the patients had a heart rate $>70$ beats per minute. The acquisition parameters were as follows: detector collimation, $64 \times 0.625 \mathrm{~mm}$; gantry rotation time, $0.27 \mathrm{~s}$; tube voltage, $120 \mathrm{kVp}$; and tube current automatic modulation. The acquisition ranged from the carina to the level of the diaphragm. A $20 \mathrm{G}$ dual-syringe power injector was used to intravenously inject $0.9 \mathrm{~mL} / \mathrm{kg}$ of contrast agent (Iopamiro, $370 \mathrm{mgI} / \mathrm{mL}$; Bracco Sine Pharma, Shanghai, China) at a $4.5-5 \mathrm{~mL} / \mathrm{s}$ flow rate, followed by $40 \mathrm{~mL}$ saline flush at the same flow rate.

Immediately after coronary CT angiography, $0.5 \mathrm{~mL} / \mathrm{kg}$ of iodinated contrast medium (Iopamiro, $370 \mathrm{mgI} / \mathrm{mL}$; Bracco Sine Pharma, Shanghai, China) was injected at 


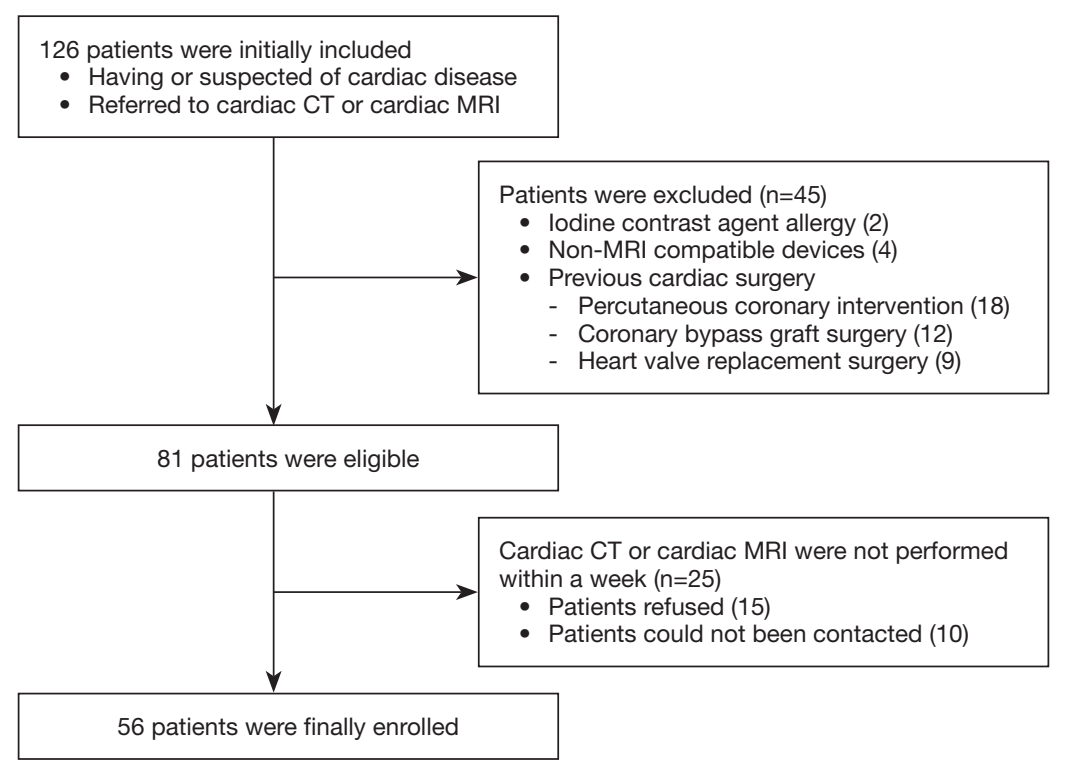

Figure 1 The flowchart shows study population enrollment. CT, computed tomography; MRI, magnetic resonance imaging.

$2.5-3 \mathrm{~mL} / \mathrm{s}$ flow rate. In total, $1.4 \mathrm{~mL} / \mathrm{kg}$ of iodinated contrast medium, with the maximum dose of contrast medium not exceeding $100 \mathrm{~mL}$, was injected $(9,13)$. CTLIE images were obtained 7 min after coronary CT angiography using the prospective axial scanning mode. The scanning parameters were the same as those of the coronary CT angiography imaging. The effective radiation dose (ED) of CT was calculated as follows: dose-length product $(\mathrm{DLP}) \times$ conversion factor of 0.014 (14).

\section{Cardiac MRI examination}

Cardiac MRI was performed using a $3 \mathrm{~T}$ whole-body scanner (MAGNETOM Skyra, Siemens Healthineers, Erlangen, Germany), with an 18-element body matrix coil and a 32-element spine array coil. A bolus of gadolinium contrast agent (Gadobenate Dimeglumine, Beijing BEILU Pharmaceutical Co, Ltd, Peking, China) was injected at a $0.05 \mathrm{mmol} / \mathrm{kg}$ dose and at a $4 \mathrm{~mL} / \mathrm{s}$ flow rate for obtaining first pass perfusion images. Then $0.1 \mathrm{mmol} / \mathrm{kg}$ gadolinium contrast agent was immediately injected at a $1 \mathrm{~mL} / \mathrm{s}$ flow rate. MRI-LGE images were obtained 10 minutes after contrast was injected with a $2 \mathrm{D}$ phase-sensitive inversionrecovery (PSIR) gradient-echo pulse sequence. The image acquisition parameters were as follows: repetition time (TR) $5.2 \mathrm{~ms}$, echo time (TE) $1.96 \mathrm{~ms}$, flip angle (FA) 20 degrees, and a voxel size of $1.4 \mathrm{~mm} \times 1.4 \mathrm{~mm} \times 8.0 \mathrm{~mm}$. Native and 15 minute postcontrast $\mathrm{T} 1$ mapping data were scanned in identical imaging locations with 3 short-axis slices (at apical, mid, and basal portions of the left ventricle), using a modified Look-Lockers inversion recovery (MOLLI) sequence. The image acquisition parameters included the following: TR $2.7 \mathrm{~ms}$, TE $1.12 \mathrm{~ms}$, FA 20 degrees, and a voxel size of $1.4 \mathrm{~mm} \times 1.4 \mathrm{~mm} \times 8.0 \mathrm{~mm}$. MRI-ECV was semiautomatically measured using dedicated cardiac MRI software CVI42 (Version 5.3, Circle Cardiovascular Imaging, Calgary, Canada) by contouring the endocardium and epicardium on the MRI-ECV maps.

\section{Image analysis}

All CT images were transferred to a commercial spectral workstation (IntelliSpace Portal Version 10.0, Philips Healthcare).

On CT-LIE images, the iodine density map was applied to the short-axis plane with an $8 \mathrm{~mm}$ slice thickness and no gap. Three short-axis slices (apical, mid, and basal left ventricle) from CT-LIE images were matched with the T1 map images from the cardiac MRI. Regions of interest (ROIs) were manually drawn in each segment of the myocardium according to the American Heart Association's 16-segment model of the left ventricle, with the extreme edges of the myocardium being avoided $(15,16)$. A circular ROI was placed in the blood pool to avoid papillary muscle (Figure 2). After the iodine density value (in $\mathrm{mg} / \mathrm{mL}$ ) was obtained from all the ROIs, the CT-ECV per segment was 

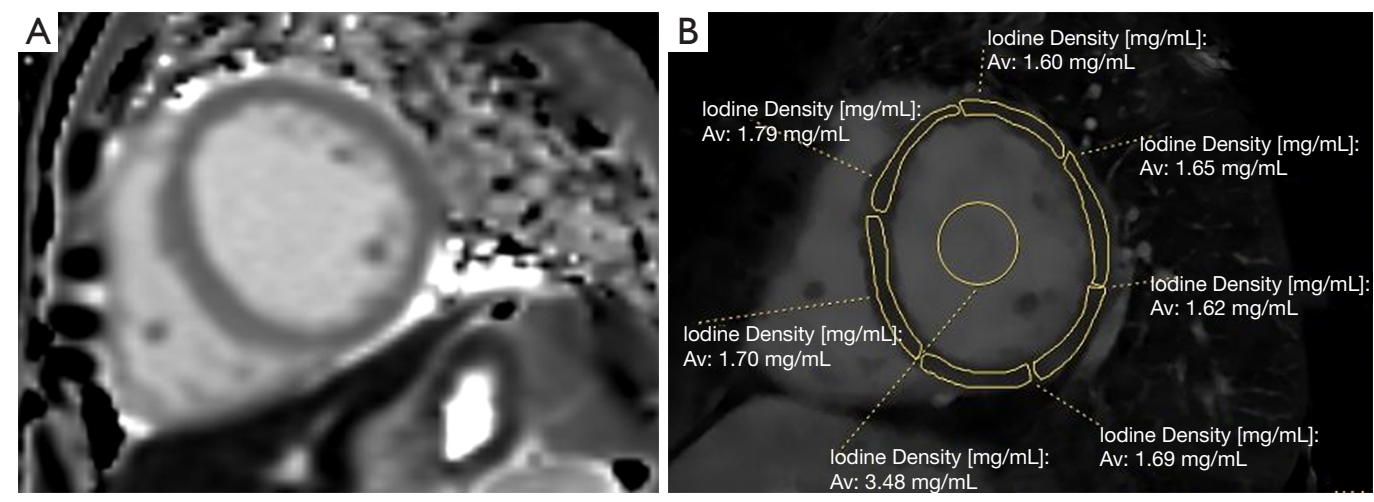

Figure 2 Example of ECV quantification. An iodine map from dual-layer spectral detector CT (B) was matched with the T1 map images from cardiac MRI (A). CT, computed tomography; ECV, extracellular volume fraction; MRI, magnetic resonance imaging.

calculated as follows (17): CT-ECV $(\%)=(1-$ hematocrit $) \times$ (iodine density in myocardium)/(iodine density in the blood pool). To calculate the reproducibility of measurements, 2 radiologists independently measured both CT-ECV and MRI-ECV.

On cardiac MRI-LGE imaging, the presence of LGE was defined as positive when myocardial enhancement was found in both short-axis and matching long-axis images (18). The LGE pattern was classified as subendocardial, transmural, midwall, epicardial, patchy, and none. Based on CT and MRI findings, we classified myocardial segments into 3 groups: ischemic LGE segments (group 1), nonischemic LGE segments (group 2) and LGE-negative segments (group 3). An ischemic pattern was defined as subendocardial or transmural myocardial delayed enhancement on MRI image corresponding to the perfusion territory of a coronary artery, and a nonischemic pattern demonstrated that myocardial delayed enhancement was not limited to a vascular territory (19-21). An ischemic segment and nonischemic segment were defined as LGE-positive segments, and an LGE-negative segment was defined as a myocardial segment without delayed enhancement. MRILGE images were independently observed by 2 experienced radiologists. Discrepancies were resolved with a third radiologist.

\section{Statistical analysis}

Continuous variables are expressed as the mean \pm standard deviation or median (interquartile range). A paired $t$-test was used to compare mean CT-ECV and MRI-ECV. An independent Mann-Whitney $U$ test was used to compare ECVs among the 3 groups. The correlations and agreement between CT-ECV and MRI-ECV on a persegment basis were evaluated using Spearman correlation and Bland-Altman analyses. ROC curve analysis, with the corresponding area under the curve (AUC), was performed to establish a threshold for LIE. Interobserver reliability for each imaging modality was tested using an intraclass correlation coefficient (ICC). A P value $<0.05$ was considered statistically significant. All statistical analyses were performed using MedCalc (version 19.6.4) and SPSS (version 25, IBM corporation, Armonk, NY, USA) software.

\section{Results}

\section{Baseline characteristics}

Based on the inclusion and exclusion criteria, a total of 56 patients ( 32 males and 24 females; mean age, $52 \pm 16$ years) were enrolled in our study and underwent both CT-LIE and MRI-LGE within a week. The patient characteristics are presented in Table 1.

Among the 56 patients, 10 patients had ischemic LGE, 28 patients had nonischemic LGE, and 18 patients had no LGE on cardiac MRI. Nonischemic patients included those with dilated cardiomyopathy $(\mathrm{n}=12)$, hypertrophic cardiomyopathy $(n=2)$, hypertensive cardiomyopathy $(n=4)$, arrhythmogenic cardiomyopathy $(\mathrm{n}=2)$, noncompaction of left ventricular myocardium $(\mathrm{n}=2)$, connective tissue disease $(\mathrm{n}=2)$, peripartum cardiomyopathy $(\mathrm{n}=1)$, and unknown conditions $(\mathrm{n}=3)$.

Among the 56 patients, 896 segments were analyzed; of these, 302 segments had LGE, with 73 segments in group 
Table 1 Patient demographics

\begin{tabular}{lc}
\hline Characteristic & Value \\
\hline Male/female & $32 / 24$ \\
Age (years) & $52.1 \pm 16.5$ \\
Body mass index $\left(\mathrm{kg} / \mathrm{m}^{2}\right)$ & $24.0 \pm 4.0$ \\
Hematocrit (\%) & $41.1 \pm 5.3$ \\
Diabetes & $23(41.1)$ \\
Hypertension & $25(44.6)$ \\
Hyperlipidemia & $10(17.9)$ \\
Smoking & $15(26.8)$ \\
\hline
\end{tabular}

Data are expressed as the mean \pm standard derivation or $\mathrm{N}(\%)$.

Table 2 Parameters for myocardial delayed iodine enhancement imaging

\begin{tabular}{lc}
\hline Parameter & Value \\
\hline Scan mode & Prospective electrocardiogram gating \\
Peak tube voltage $(\mathrm{kVp})$ & 120 \\
Tube current $(\mathrm{mA})$ & $124.7 \pm 31.9$ \\
Total contrast dose $(\mathrm{mL})$ & $89.5 \pm 11.6$ \\
CTDlvol $(\mathrm{mGy})$ & $15.0 \pm 4.5$ \\
DLP $(\mathrm{mGy} \cdot \mathrm{cm})$ & $188.4 \pm 65.7$ \\
ED $(\mathrm{mSv})$ & $2.6 \pm 0.9$ \\
\hline
\end{tabular}

Data are expressed as the mean \pm standard derivation. CTDIvol, volume computed tomography dose index; DLP, dose-length product; ED, effective radiation dose.

1 and 229 segments in group 2; meanwhile, 594 segments group 3 showed no LGE. On MRI-LGE imaging, 18\% $(55 / 302)$ of segments showed a subendocardial pattern, $13 \%(38 / 302)$ showed a transmural pattern, 26\% (77/302) showed a midwall pattern, $5 \%(16 / 302)$ showed an epicardial pattern, and 38\% (116/302) showed a patchy pattern. The median DLP and mean ED of the LIE images were $188.4 \pm 65.7 \mathrm{mGy} \cdot \mathrm{cm}$ and $2.6 \pm 0.9 \mathrm{mSv}$, respectively. The scan parameters are shown in Table 2.

\section{Myocardial ECV in per-subject analysis}

In a per-subject analysis, the myocardial ECV of CT imaging was $28.7 \%(26.5-31.9 \%)$, and that of MRI imaging was $28.4 \%$ (26.6-32.2\%). There was no significant difference between CT-ECV and MRI-ECV $(\mathrm{P}=0.383)$.

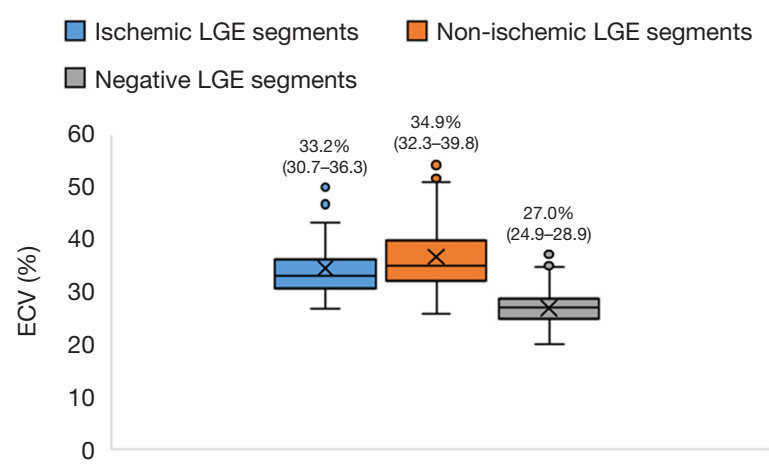

Figure 3 The mean CT-ECV values on ischemic LGE myocardial segments (group 1), nonischemic LGE myocardial segments (group 2), and LGE-negative myocardial segments (group 3). CT-ECV in group 3 was significantly lower than that in group 1 and group 2 (all $\mathrm{P}$ values <0.001). CT, computed tomography; ECV, extracellular volume fraction; LGE, late gadolinium enhancement.

Interobserver agreement for ECV on CT imaging and MRI was excellent (ICC $=0.889$ and 0.904 , respectively).

\section{Myocardial ECV in per-segment analysis}

In a per-segment analysis, the CT-ECV value of subendocardial, transmural, midwall, epicardial, and patchy pattern was $35.9 \%$ (27.5-50.1\%), 40.7\% (29.5-54.3\%), $34.4 \%$ (26.9-47.4\%), 38.8\% (30.1-50.4\%), and 37.0\% (26.0$50.9 \%)$, respectively. The CT-ECV in group $1(33.2 \% ; 30.7-$ $36.3 \%)$ and group $2(34.9 \% ; 32.3-39.8 \%)$ was significantly higher than that of group $3(27.0 \% ; 24.9-28.9 \%$; all $\mathrm{P}$ values $<0.001)$. These results are summarized in Figure 3.

Good correlations were seen between CT-ECV and MRI-ECV measurements for group $1(\mathrm{r}=0.920)$, group 2 $(\mathrm{r}=0.936)$, and group $3(\mathrm{r}=0.799$; all $\mathrm{P}$ values $<0.001)$. BlandAltman analysis between CT-ECV and MRI-ECV showed a small bias (group 1: $-2.1 \%, 95 \%$ limits of agreement $-11.3-7.1 \%$; group $2:-0.6 \%, 95 \%$ limits of agreement $-13.1-11.9 \%$; group $3: 1.0 \%, 95 \%$ limits of agreement $-12.7-14.7 \%$; Figures 4,5 ).

From ROC curve analysis, CT-ECV differentiated between LGE-positive and LGE-negative segments, with an $83.1 \%$ sensitivity and a $93.3 \%$ specificity at a cutoff of $31 \%$; the area under the curve of ROC was 0.957 . The same analysis also suggested that CT-ECV could differentiate between ischemic LGE myocardial segments and LGE-negative myocardial segments (sensitivity 93.2\%; 
A

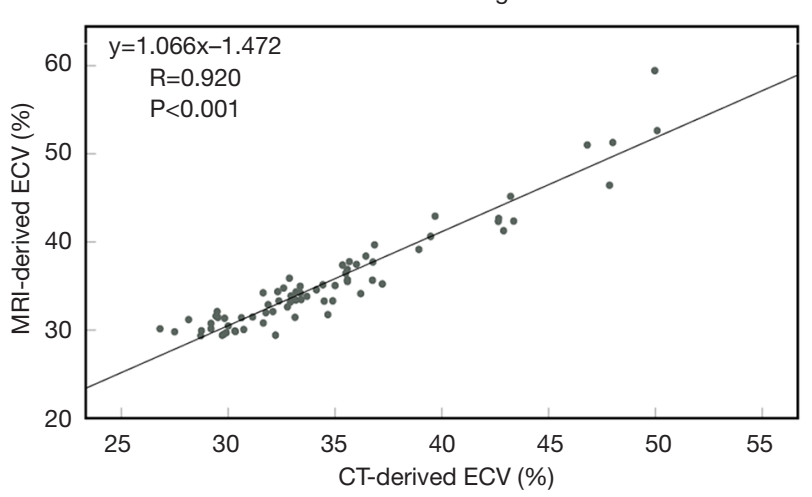

C

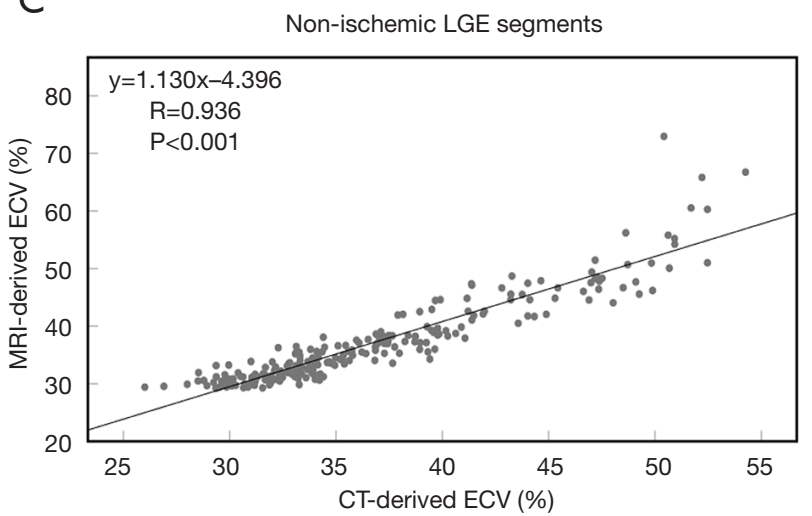

$\mathrm{E}$

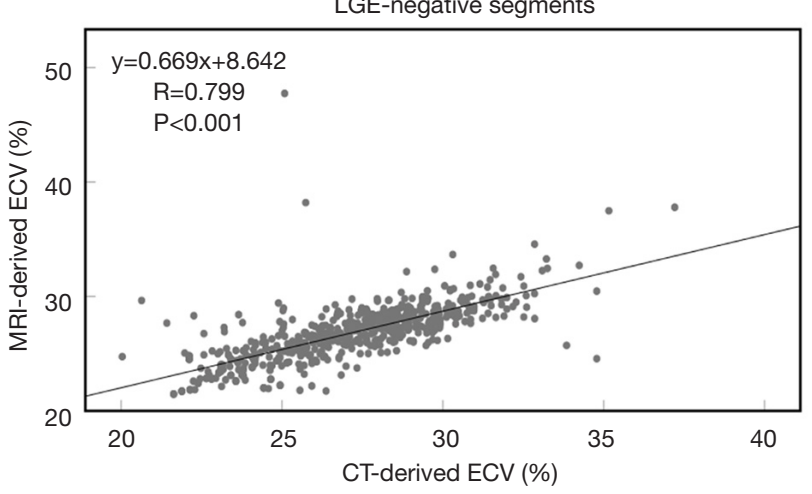

B

Ischemic LGE segments
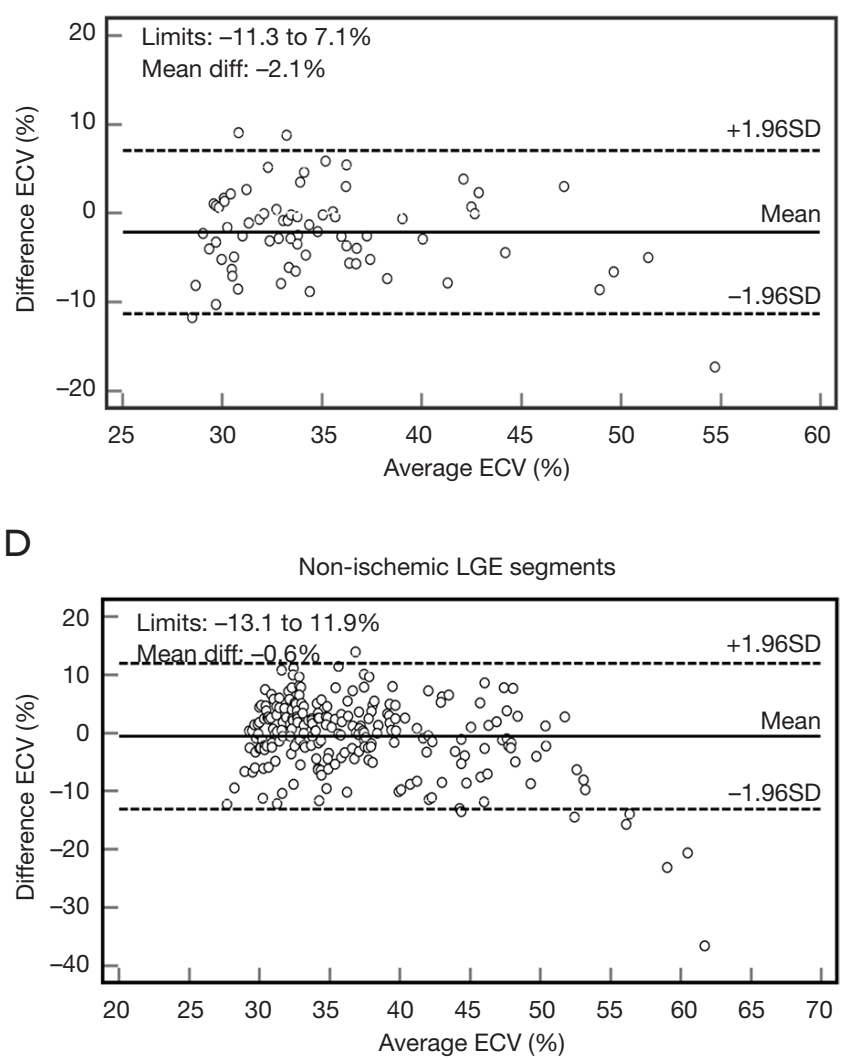

$\mathrm{F}$

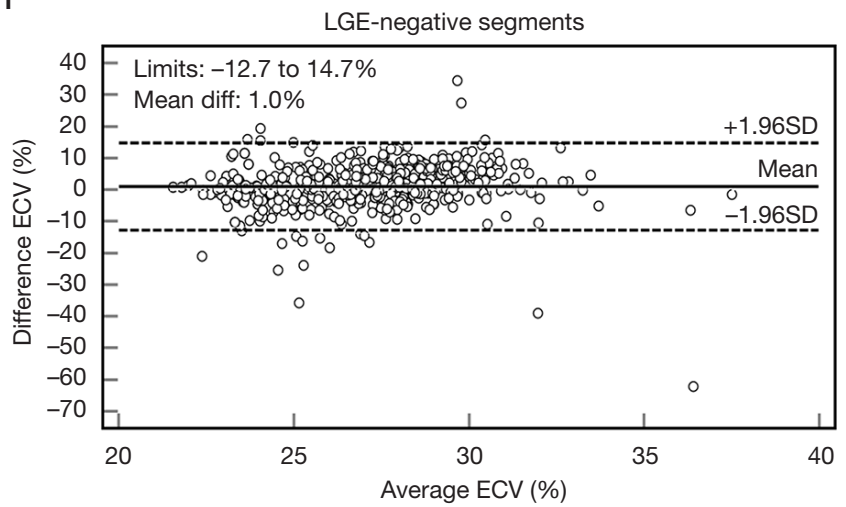

Figure 4 Scatter plots and Bland-Altman plots. The scatter plots show the results of correlation (A, C, and E) and Bland-Altman plots show the results of agreement (B, D, and F) for comparisons between CT- and MRI-derived ECV on ischemic LGE segments, nonischemic LGE segments, and LGE-negative myocardial segments. CT, computed tomography; ECV, extracellular volume fraction; LGE, late gadolinium enhancement; MRI, magnetic resonance imaging. 

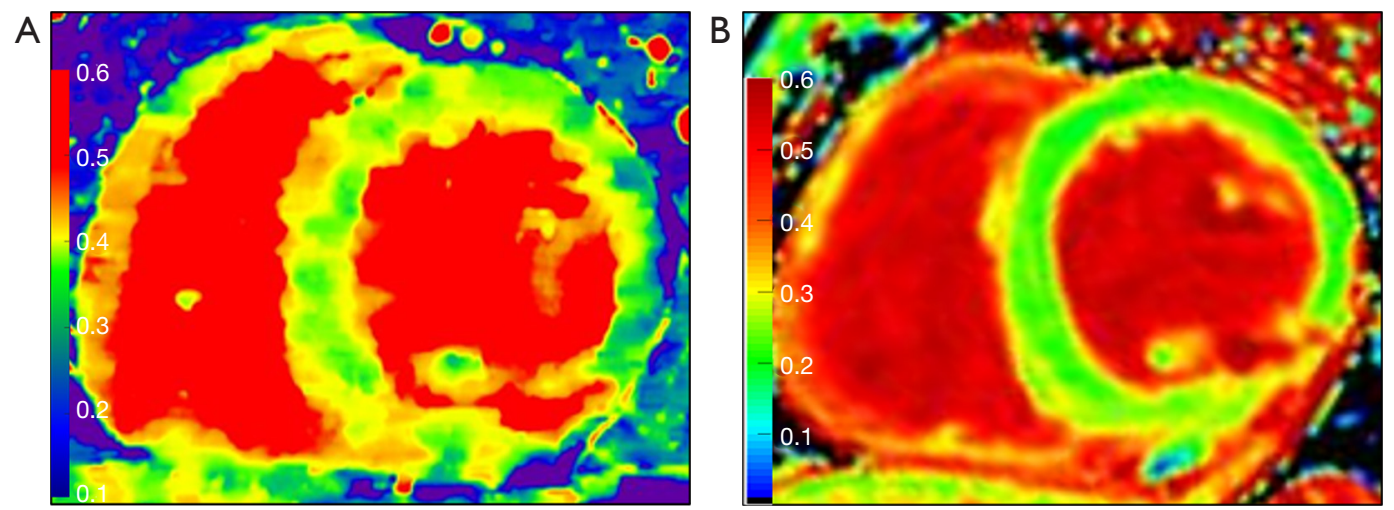

Figure 5 Example of ECV maps. Comparison of a CT-derived ECV map (A) and an MRI-derived ECV map (B) in a 50-year-old man. Increased ECV values were observed on both CT-ECV and MRI-ECV of the basal inferolateral LV myocardium (ECV =53\% and $50 \%$, respectively). CT, computed tomography; ECV, extracellular volume; LV, left ventricle; MRI, magnetic resonance imaging.
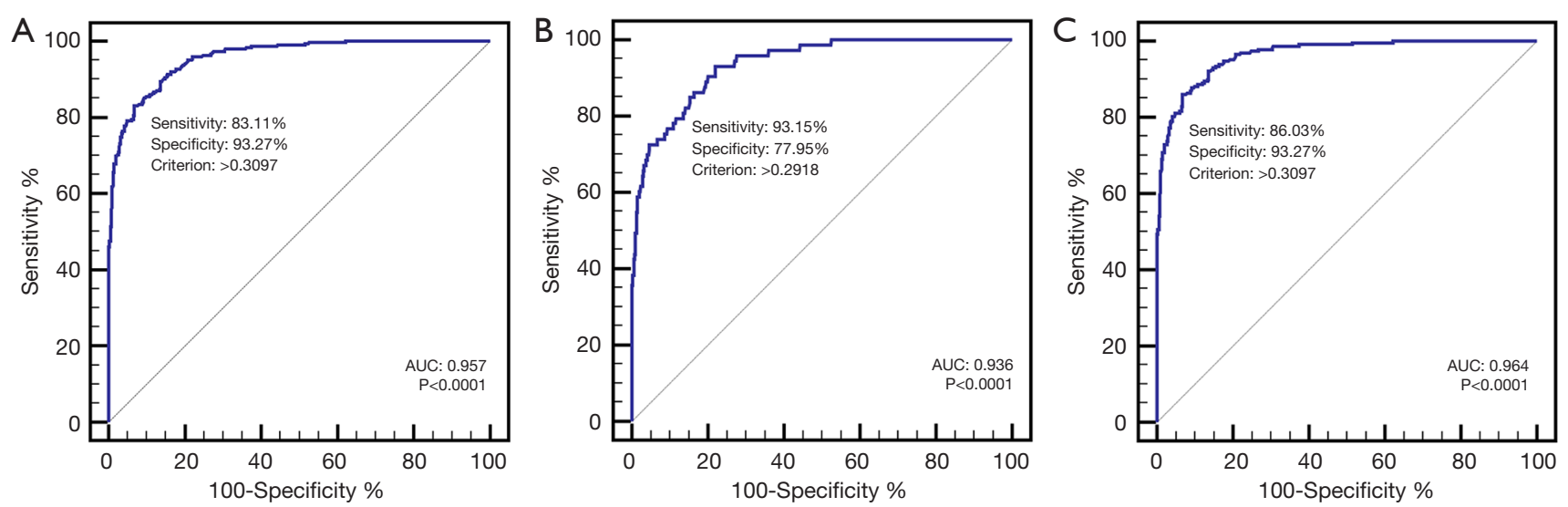

Figure 6 ROC curves. Results from ROC curve analysis (with a corresponding AUC) to differentiate LGE-positive and LGE-negative myocardial segments (A). Results from ROC curve analysis (with a corresponding AUC) to differentiate ischemic LGE myocardial segments and LGE-negative myocardial segments (B), and to differentiate non-ischemic LGE myocardial segments and LGE-negative myocardial segments (C). AUC, area under the curve; LGE, late gadolinium enhancement; ROC, receiver operating characteristic.

specificity $78.0 \%$; AUC 0.936 ) and between nonischemic LGE myocardial segments and LGE-negative myocardial segments (sensitivity $86.0 \%$; specificity $93.3 \%$; AUC 0.964; Figure 6).

\section{Discussion}

This study showed the feasibility of quantifying myocardial ECV using dual-layer spectral detector CT with the iodine density maps. First, our results demonstrated a strong correlation with a small bias between segmental CT-ECV and MRI-ECV. Second, our results suggested that an ECV $>31 \%$ could differentiate between LGE-positive and LGE- negative segments.

There are two methods to calculate CT-ECV: the subtraction-derived method and the iodine density-derived method. The subtraction-derived method requires the acquisition of precontrast and delayed images to obtain $\mathrm{ECV}$ value. It is relatively difficult to perfectly match these 2 images because of the movement of the heart and the diaphragm. Misalignment may cause deviation of ECV value. The iodine density-derived method of calculating ECV has notable advantages over the subtraction-derived method. ECV derived from iodine density images needs only a single delayed acquisition. This method does not require image co-registration, enabling precise evaluation 
of ECV. Emoto et al. (10) evaluated CT-derived ECV quantification between the subtraction-derived method and the iodine density-derived method using dual-layer spectral detector CT, and compared the quantification outcomes to those determined by MRI. The evaluation revealed that the iodine density-derived method yielded more accurate myocardial ECV quantification than did the subtractionderived method and had a strong correlation with MRI. Our results also confirmed a strong correlation of CT-ECV using iodine density maps from dual-layer spectral detector CT, with MRI-ECV demonstrating a small bias.

Some recent studies have investigated the feasibility of CT-ECV in different cardiac diseases, including cardiac amyloidosis (22), hypertrophic cardiomyopathy (23), dilated cardiomyopathy (24), heart failure (7), and in hemodialysis patients (25). Ohta et al. (24) evaluated CT-ECV in both nonischemic dilated cardiomyopathy patients and normal participants, and defined a threshold (a cutoff of 28.82\%) to distinguish healthy myocardial tissue from dilated cardiomyopathy patients. However, this study did not include ischemic cardiomyopathy patients, and the ECV results were not compared with MRI-ECV. Abadia et al. (12) investigated the CT-ECV of healthy myocardium, nonischemic cardiomyopathy, and ischemic cardiomyopathy, and set a threshold (a cutoff of $29.5 \%$ ) to discriminate healthy participants from cardiomyopathy patients, but the CT-ECV was not compared with MRI-ECV in this work. In our study, we found a strong correlation of CTECV with MRI-ECV and set a cutoff value (31\%) with a $83.1 \%$ sensitivity in differentiating between LGE-positive and LGE-negative segments. However, approximately $17 \%$ of LGE-positive patients showed false-negative results on CT image. Possible explanations for this are the misdiagnosis of small thin fibrosis. In the case of a subendocardial enhancement, the signal intensity in the delayed enhancement area is similar to that from the adjacent blood pool; therefore, it is difficult to differentiate subendocardial enhancement from the blood pool. Subepicardial enhancement and midwall enhancement are also misdiagnosed in iodine density images due to a lower contrast to noise ratio compared to other LGE patterns. Thus, it is necessary to further perform MRI examination for these small LGE patterns.

To date, a standard contrast injection protocol has not been established for ECV quantification on cardiac CT. Two common contrast injection methods were used in the present study. Lee et al. (16) chose a single contrast injection protocol with an infusion of $1.8 \mathrm{~mL} / \mathrm{kg}$ of body weight to obtain coronary CT angiography and CT delayed enhancement imaging. Ohta et al. (9) used a 2-contrast injection protocol: one with an infusion of $0.9 \mathrm{~mL} / \mathrm{kg}$ of body weight for coronary CT angiography followed by another injection of $0.5 \mathrm{~mL} / \mathrm{kg}$ of body weight immediately after coronary CT angiography. To obtain good contrast between myocardial tissue and the left ventricular cavity on CT delayed enhancement images, the amount of iodine contrast medium used for calculating ECV has ranged from 120 to $147 \mathrm{~mL}$ in some previous studies $(23,26,27)$. These relatively big volumes of iodine contrast in a single contrast injection protocol may result in a high density of contrast material in the right heart cavities, which may cause streak artifacts affecting the lumen evaluation of the proximal right coronary artery. Thus, the contrast medium injection in our study was performed twice in the CT protocol in order to better assess the coronary artery lumen while evaluating the myocardium.

Several limitations must be considered in this study: first, cardiac CT-ECV measurements were compared with cardiac MRI findings rather than with histologic findings because the latter is hard to obtain, and a previous study has reported that CT-ECV correlated well with histologic quantification and MRI-ECV for detecting myocardial fibrosis (8). Second, this study did not compare CT-ECV values based on the percentage of LGE in the segment. Further studies are needed to explore the feasibility of CT$\mathrm{ECV}$ in different delayed enhancement patterns, which is helpful for clinical management. Third, a relatively high contrast volume was used to obtain CT-LIE images for ECV measurements (a total of $89.5 \mathrm{~mL}$ contrast dose in our study), and further studies need to investigate the feasibility of ECV quantification with a low contrast volume using dual-layer spectral CT for reducing the risk of contrastinduced nephropathy.

\section{Conclusions}

ECV derived from CT showed good correlation and agreement with MRI findings, and CT-ECV provided high diagnostic accuracy to allow discrimination between LGE-positive and LGE-negative segments. Thus, cardiac CT might be a suitable noninvasive imaging technique for myocardial ECV quantification.

\section{Acknowledgments}

The authors would like to thank Wei Han for his assistance 
with statistical analysis.

Funding: This work was supported by the National Natural Science Foundation of China (grant numbers 81873891 and 82020108018), the Fundamental Research Funds for the Central Universities (grant number 3332020008), and the CAMS Innovation Fund for Medical Sciences (grant number 2020-I2M-C-T-B-034).

\section{Footnote}

Conflicts of Interest: All authors have completed the ICMJE uniform disclosure form (available at https://dx.doi. org/10.21037/qims-21-344). The authors have no conflicts of interest to declare.

Ethical Statement: The authors are accountable for all aspects of the work in ensuring that questions related to the accuracy or integrity of any part of the work are appropriately investigated and resolved. The study was conducted in accordance with the Declaration of Helsinki (as revised in 2013). This study was approved by Institutional Committee of Ethics of Peking Union Medical College Hospital. All participants signed written informed consent before undergoing cardiac CT and MRI scans.

Open Access Statement: This is an Open Access article distributed in accordance with the Creative Commons Attribution-NonCommercial-NoDerivs 4.0 International License (CC BY-NC-ND 4.0), which permits the noncommercial replication and distribution of the article with the strict proviso that no changes or edits are made and the original work is properly cited (including links to both the formal publication through the relevant DOI and the license). See: https://creativecommons.org/licenses/by-nc-nd/4.0/.

\section{References}

1. Kammerlander AA, Marzluf BA, Zotter-Tufaro C, Aschauer S, Duca F, Bachmann A, Knechtelsdorfer K, Wiesinger M, Pfaffenberger S, Greiser A, Lang IM, Bonderman D, Mascherbauer J. T1 Mapping by CMR Imaging: From Histological Validation to Clinical Implication. JACC Cardiovasc Imaging 2016;9:14-23.

2. Ugander M, Oki AJ, Hsu LY, Kellman P, Greiser A, Aletras AH, Sibley CT, Chen MY, Bandettini WP, Arai AE. Extracellular volume imaging by magnetic resonance imaging provides insights into overt and sub-clinical myocardial pathology. Eur Heart J 2012;33:1268-78.
3. Messroghli DR, Moon JC, Ferreira VM, GrosseWortmann L, He T, Kellman P, Mascherbauer J, Nezafat R, Salerno M, Schelbert EB, Taylor AJ, Thompson R, Ugander M, van Heeswijk RB, Friedrich MG. Clinical recommendations for cardiovascular magnetic resonance mapping of $\mathrm{T} 1, \mathrm{~T} 2, \mathrm{~T} 2{ }^{*}$ and extracellular volume: A consensus statement by the Society for Cardiovascular Magnetic Resonance (SCMR) endorsed by the European Association for Cardiovascular Imaging (EACVI). J Cardiovasc Magn Reson 2017;19:75.

4. Zhou Z, Wang R, Wang H, Liu Y, Lu D, Sun Z, Yang G, Xu L. Myocardial extracellular volume fraction quantification in an animal model of the doxorubicininduced myocardial fibrosis: a synthetic hematocrit method using 3T cardiac magnetic resonance. Quant Imaging Med Surg 2021;11:510-20.

5. Haaf P, Garg P, Messroghli DR, Broadbent DA, Greenwood JP, Plein S. Cardiac T1 Mapping and Extracellular Volume (ECV) in clinical practice: a comprehensive review. J Cardiovasc Magn Reson 2016;18:89.

6. Thuny F, Lovric D, Schnell F, Bergerot C, Ernande L, Cottin V, Derumeaux G, Croisille P. Quantification of myocardial extracellular volume fraction with cardiac $M R$ imaging for early detection of left ventricle involvement in systemic sclerosis. Radiology 2014;271:373-80.

7. Wang R, Liu X, Schoepf UJ, van Assen M, Alimohamed I, Griffith LP, Luo T, Sun Z, Fan Z, Xu L. Extracellular volume quantitation using dual-energy CT in patients with heart failure: Comparison with $3 \mathrm{~T}$ cardiac MR. Int J Cardiol 2018;268:236-40.

8. Bandula S, White SK, Flett AS, Lawrence D, Pugliese F, Ashworth MT, Punwani S, Taylor SA, Moon JC. Measurement of myocardial extracellular volume fraction by using equilibrium contrast-enhanced CT: validation against histologic findings. Radiology 2013;269:396-403.

9. Ohta Y, Kishimoto J, Kitao S, Yunaga H, MukaiYatagai N, Fujii S, Yamamoto K, Fukuda T, Ogawa T. Investigation of myocardial extracellular volume fraction in heart failure patients using iodine map with rapid$\mathrm{kV}$ switching dual-energy CT: Segmental comparison with MRI T1 mapping. J Cardiovasc Comput Tomogr 2020;14:349-55.

10. Emoto T, Oda S, Kidoh M, Nakaura T, Nagayama $Y$, Sakabe D, Kakei K, Goto M, Funama Y, Hatemura M, Takashio S, Kaikita K, Tsujita K, Ikeda O. Myocardial Extracellular Volume Quantification Using Cardiac 
Computed Tomography: A Comparison of the Dualenergy Iodine Method and the Standard Subtraction Method. Acad Radiol 2021;28:e119-26.

11. Emoto T, Kidoh M, Oda S, Nakaura T, Nagayama Y, Sasao A, Funama Y, Araki S, Takashio S, Sakamoto K, Yamamoto E, Kaikita K, Tsujita K, Yamashita Y. Myocardial extracellular volume quantification in cardiac CT: comparison of the effects of two different iterative reconstruction algorithms with $\mathrm{MRI}$ as a reference standard. Eur Radiol 2020;30:691-701.

12. Abadia AF, van Assen M, Martin SS, Vingiani V, Griffith LP, Giovagnoli DA, Bauer MJ, Schoepf UJ. Myocardial extracellular volume fraction to differentiate healthy from cardiomyopathic myocardium using dualsource dual-energy CT. J Cardiovasc Comput Tomogr 2020;14:162-7.

13. Ohta Y, Kitao S, Yunaga H, Fujii S, Mukai N, Yamamoto K, Ogawa T. Myocardial Delayed Enhancement CT for the Evaluation of Heart Failure: Comparison to MRI. Radiology 2018;288:682-91.

14. Halliburton SS, Abbara S, Chen MY, Gentry R, Mahesh M, Raff GL, Shaw LJ, Hausleiter J; Society of Cardiovascular Computed Tomography. SCCT guidelines on radiation dose and dose-optimization strategies in cardiovascular CT. J Cardiovasc Comput Tomogr 2011;5:198-224.

15. Cerqueira MD, Weissman NJ, Dilsizian V, Jacobs AK, Kaul S, Laskey WK, Pennell DJ, Rumberger JA, Ryan T, Verani MS; American Heart Association Writing Group on Myocardial Segmentation and Registration for Cardiac Imaging. Standardized myocardial segmentation and nomenclature for tomographic imaging of the heart. A statement for healthcare professionals from the Cardiac Imaging Committee of the Council on Clinical Cardiology of the American Heart Association. Circulation 2002;105:539-42.

16. Lee HJ, Im DJ, Youn JC, Chang S, Suh YJ, Hong YJ, Kim YJ, Hur J, Choi BW. Myocardial Extracellular Volume Fraction with Dual-Energy Equilibrium Contrastenhanced Cardiac CT in Nonischemic Cardiomyopathy: A Prospective Comparison with Cardiac MR Imaging. Radiology 2016;280:49-57.

17. Oda S, Emoto T, Nakaura T, Kidoh M, Utsunomiya D, Funama Y, Nagayama Y, Takashio S, Ueda M, Yamashita T, Tsujita K, Ando Y, Yamashita Y. Myocardial Late Iodine Enhancement and Extracellular Volume Quantification with Dual-Layer Spectral Detector DualEnergy Cardiac CT. Radiol Cardiothorac Imaging 2019;1:e180003.

18. Wang Y, Wang Q, Cao J, Li X, Lin L, Chen W, Wang YN, Jin ZY. Cardiovascular Magnetic Resonance Mapping and Strain Assessment for the Diagnosis of Cardiac Involvement in Idiopathic Inflammatory Myopathy Patients With Preserved Left Ventricular Ejection Fraction. J Thorac Imaging 2021;36:254-61.

19. Cummings KW, Bhalla S, Javidan-Nejad C, Bierhals AJ, Gutierrez FR, Woodard PK. A pattern-based approach to assessment of delayed enhancement in nonischemic cardiomyopathy at MR imaging. Radiographics 2009;29:89-103.

20. Mahrholdt H, Wagner A, Judd RM, Sechtem U, Kim RJ. Delayed enhancement cardiovascular magnetic resonance assessment of non-ischaemic cardiomyopathies. Eur Heart J 2005;26:1461-74.

21. Rajiah P, Desai MY, Kwon D, Flamm SD. MR imaging of myocardial infarction. Radiographics 2013;33:1383-412.

22. Treibel TA, Bandula S, Fontana M, White SK, Gilbertson JA, Herrey AS, Gillmore JD, Punwani S, Hawkins PN, Taylor SA, Moon JC. Extracellular volume quantification by dynamic equilibrium cardiac computed tomography in cardiac amyloidosis. J Cardiovasc Comput Tomogr 2015;9:585-92.

23. Mirelis JG, Sánchez-González J, Zorio E, Ripoll-Vera T, Salguero-Bodes R, Filgueiras-Rama D, GonzálezLópez E, Gallego-Delgado M, Fernández-Jiménez R, Soleto MJ, Núñez J, Pizarro G, Sanz J, Fuster V, GarcíaPavía P, Ibáñez B. Myocardial Extracellular Volume Is Not Associated With Malignant Ventricular Arrhythmias in High-risk Hypertrophic Cardiomyopathy. Rev Esp Cardiol (Engl Ed) 2017;70:933-40.

24. Ohta Y, Kitao S, Yunaga H, Watanabe T, Mukai-Yatagai N, Kishimoto J, Yamamoto K, Ogawa T. Quantitative evaluation of non-ischemic dilated cardiomyopathy by late iodine enhancement using rapid $\mathrm{kV}$ switching dual-energy computed tomography: A feasibility study. J Cardiovasc Comput Tomogr 2019;13:148-56.

25. Yamada A, Kitagawa K, Nakamura S, Takafuji M, Goto Y, Okamoto R, Dohi K, Sakuma H. Quantification of extracellular volume fraction by cardiac computed tomography for noninvasive assessment of myocardial fibrosis in hemodialysis patients. Sci Rep 2020;10:15367.

26. Nacif MS, Liu Y, Yao J, Liu S, Sibley CT, Summers RM, Bluemke DA. 3D left ventricular extracellular volume fraction by low-radiation dose cardiac CT: assessment of interstitial myocardial fibrosis. J Cardiovasc Comput 
Tomogr 2013;7:51-7.

27. Kurita Y, Kitagawa K, Kurobe Y, Nakamori S, Nakajima H, Dohi K, Ito M, Sakuma H. Estimation of myocardial extracellular volume fraction with cardiac CT in subjects without clinical coronary artery disease: A feasibility study. J Cardiovasc Comput Tomogr 2016;10:237-41.

Cite this article as: Liu $\mathrm{P}, \mathrm{Lin} \mathrm{L}, \mathrm{Xu} \mathrm{C}, \operatorname{Han} \mathrm{Y}$, Lin $\mathrm{X}$, Hou Y, Lu X, Vembar M, Jin Z, Wang Y. Quantitative analysis of late iodine enhancement using dual-layer spectral detector computed tomography: comparison with magnetic resonance imaging. Quant Imaging Med Surg 2022;12(1):310-320. doi: 10.21037/qims-21-344 